\title{
Permeability Measurements of Thin Film Using a Flexible Microstrip Line-Type Probe Up To $40 \mathrm{GHz}$
}

\author{
S. Yabukami, K. Kusunoki*, H. Uetake, H. Yamada *, T. Ozawa, R. Utsumi**, T. Moriizumi***, \\ and Y. Shimada**** \\ Tohoku Gakuin University, 1-13-1 Chuo, Tagajo 985-8537, Japan \\ *Sendai National College of Technology, 48 Nodayama, Medeshima-Shiote, Natori 981-1239, Japan \\ **Toei Scientific Industrial Company Ltd., 1-101-60 Medeshimadai, Natori 981-1251, Japan \\ ${ }^{* * *}$ Candox Systems Inc., 15-21 Oshiage-Cho, Gyoda 361-0045, Japan \\ $* * * *$ Graduate School of Engineering, Tohoku University, 6-6-05 Aramaki Aza Aoba, Aoba -ku, Sendai 980-8579, Japan
}

\begin{abstract}
A highly sensitive probe based on the skin effect was developed to measure thin film permeability. A new microstrip-line-type probe on a flexible substrate was fabricated and placed in contact with a magnetic thin film. The probe enhanced the signal-to-noise ratio and broadband measurement. The permeability of amorphous CoNbZr film $(25 \mathrm{~mm} \times 25 \mathrm{~mm}, 5 \mathrm{~nm}$ thick $)$ and that of $\mathrm{CoFeB}$ film $(45 \mathrm{~mm} \times 25 \mathrm{~mm}, 0.5 \mu \mathrm{m}$ thick $)$ were optimized. The measured values were in rough agreement with theoretical values based on the Landau-Lifshitz-Gilbert equation and eddy current generation up to $40 \mathrm{GHz}$.
\end{abstract}

Key words: flexible microstrip-line-type probe, skin effect, permeability, very thin film

\section{Introduction}

High frequency permeability of very thin magnetic films is important because sensing devices and spintronic devices are fabricated from such film (film thickness of less than $10 \mathrm{~nm}$ ). However, almost all permeameters ${ }^{1)-3)}$ require a special sample, usually no more than several millimeters in width and more than $100 \mathrm{~nm}$ in thickness. Exceedingly few permeameters can measure thin film over $30 \mathrm{GHz}$ continuously.

As previously reported, we have developed a microstrip-line-type probe ${ }^{4}$. The probe is not always applicable for evaluation of very thin film, such as that less than $10 \mathrm{~nm}$ in thickness, because of the low signal-to-noise ratio. Subsequently, we also developed a straight microstrip-line-type probe on a flexible polyimide substrate ${ }^{5}$. However, the probe resonates at a frequency of over $7 \mathrm{GHz}$ because of impedance mismatch and is not applicable for large samples.

In the present study, we developed a new probe composed of a straight microstrip line on a flexible substrate, the microstrip line sloping to meet the lead line at either end. The flexibility of the probe enables contact between it and a magnetic thin film, which enhances the signal-to-noise ratio. The probe, including the microstrip line and lead lines, has a characteristic impedance of around $50 \mathrm{ohm}$. As a result, the permeability of very thin film was evaluated up to 40 GHz.

\section{Experimental setup}

\subsection{A new probe and system setup}

Fig. 1(a) shows a schematic diagram of the probe. Figs. 1(b) and (c) show a photograph of the probe. The new probe is composed of a straight microstrip conductor ( $15 \mathrm{~mm}$ in length, $0.36 \mathrm{~mm}$ in width) on a flexible substrate (RT/duroid ${ }^{\circledR}$ 5870, $130 \mu \mathrm{m}$ in

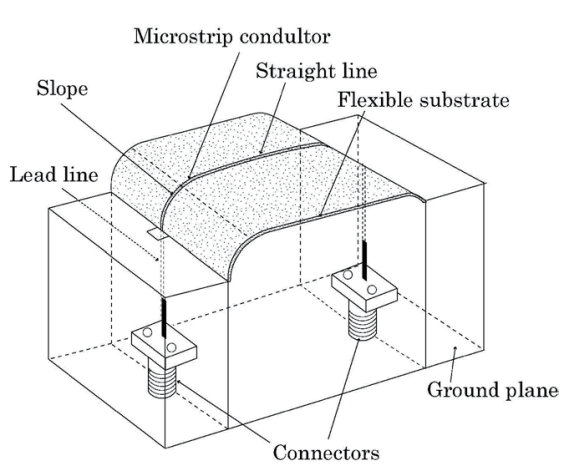

(a) oblique drawing

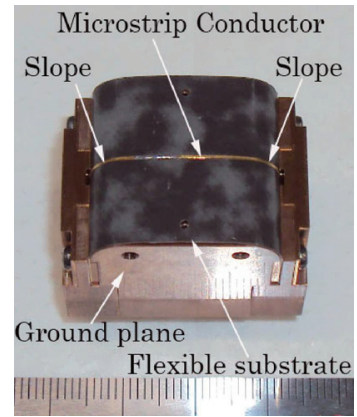

(b) top view

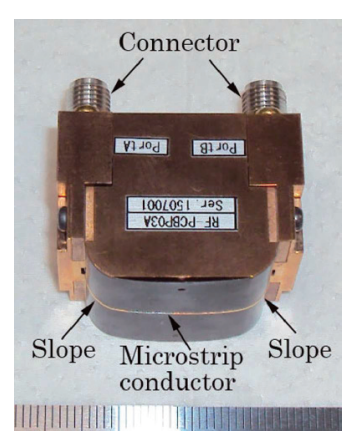

(c) side view
Fig. 1 Schematic view of probe.

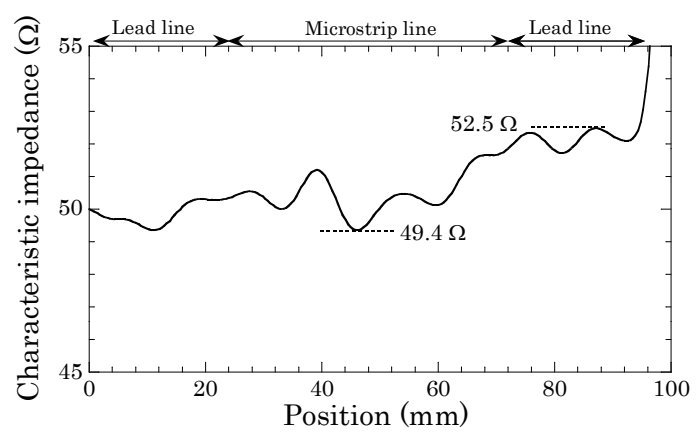

Fig. 2 Characteristic impedance of the probe obtained from time-domain reflectometry (TDR) measurements. 


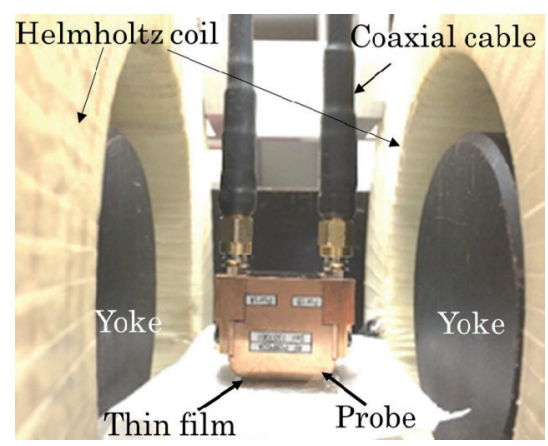

Fig. 3 Photograph of the probe and film.

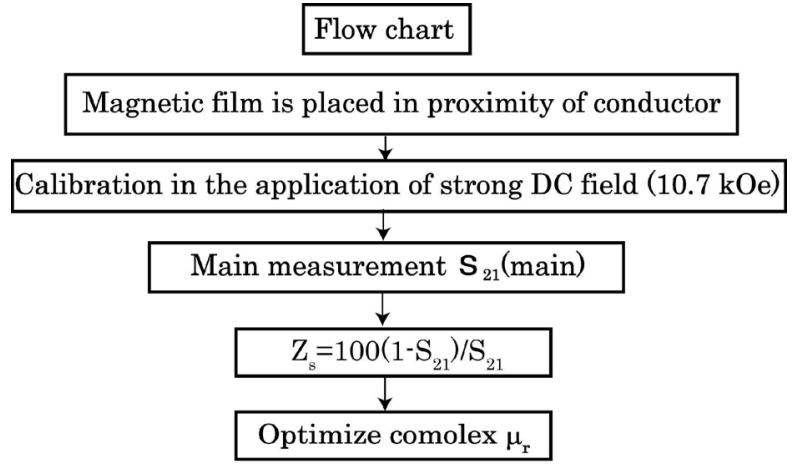

Fig. 4 Flow chart of the permeability measurements.

thickness, $\varepsilon_{r}=2.3$ ), a ground plane, lead lines, and two connectors. The microstrip line has slopes to maintain a characteristic impedance of around $50 \Omega$ and to allow close contact of a large sample with the probe. A magnetic thin film coated with photo-resist (about $8 \mu \mathrm{m}$ in thickness) is in contact with the microstrip conductor. Coaxial cables are connected to a network analyzer. The probe can be easily bent because of the flexibility of the substrate, enabling a good fit between the probe and the thin film, resulting in improved signal-to-noise ratio.

Fig. 2 shows the characteristic impedance of the probe measured by time domain reflectometry (Agilent Technologies N5227A). The characteristic impedance was $49.4 \Omega-52.5 \Omega$ along the microstrip line.

The system setup was the same as the previous works ${ }^{4), 5}$. Fig. 3 shows a photograph of the probe, a magnetic film, a Helmholtz coil, and a micrometer. The spacing between the probe and the film is adjusted by a micrometer. An Fe yoke ( $80 \mathrm{~mm}$ in diameter) is arranged around the bias coil to increase the $\mathrm{dc}$ magnetic field.

\subsection{Optimization of permeability}

Fig. 4 shows a flow chart of permeability optimization. Firstly, $S_{21}$ is calibrated by application of a strong dc field (around $850.65 \mathrm{kA} / \mathrm{m}(10.7 \mathrm{kOe})$ ) in the direction of the easy axis to saturate the magnetic film. Secondly, $S_{21}$ is measured without a strong dc field, and then the complex impedance is calculated by equation (1)

$$
Z_{s}=100\left(1-S_{21}\right) / S_{21}
$$

The $S_{21}$ and $Z_{\mathrm{s}}$ include the multiple reflections in equation (1). Complex permeability is optimized using the Newton-Raphson method ${ }^{6}$ to take the skin effect of the magnetic film into account by using equations (2) - (4),

$$
\begin{array}{r}
Z_{s}=\frac{k_{s} \rho l}{2 w} \operatorname{coth}\left(\frac{k_{s} t}{2}\right)-\left\{\frac{k_{s}^{\prime} \rho l}{2 w} \operatorname{coth}\left(\frac{k_{s}^{\prime} t}{2}\right)\right\} \frac{1}{S_{21}} \\
k_{s}=\frac{(1+j)}{\sqrt{\frac{\rho}{\pi f \mu_{r} \mu_{0}}}} \text { (3) } \quad k_{s}^{\prime}=\frac{(1+j)}{\sqrt{\frac{\rho}{\pi f \mu_{r}^{\text {ref }} \mu_{0}}}}
\end{array}
$$

where $\rho$ is the resistivity of the film, $t$ is the film thickness, $l$ is the microstrip line length, $w$ is the width of the microstrip conductor, and $\mu_{r}{ }^{r e f}$ is relative permeability when a strong dc field of $10.7 \mathrm{kOe}$ was applied. The high frequency current induces a magnetic field in the width direction of the conductor pattern, and the magnetic field and the eddy current are localized in the skin of the magnetic film ${ }^{4), 5}$.

\section{Experimental results}

Fig. 5 shows the $\mathrm{MH}$ curves of $\mathrm{Co}_{85} \mathrm{Nb}_{12} \mathrm{Zr}_{3}$ film and $\mathrm{CoFeB}$ film. Fig. 5(a) shows the MH curve of $\mathrm{CoNbZr}$ film $(25 \mathrm{~mm} \times 25 \mathrm{~mm}$ and $5 \mathrm{~nm}$ in thickness). The CoNbZr film was deposited by $\mathrm{RF}$ sputtering. The solid line shows the MH curve of the easy axis, and the dotted line shows that of the hard axis. The resistivity of the film was about $1.52 \times 10^{-6}$ $\Omega \mathrm{m}(152 \mu \Omega \mathrm{cm})$, which was slightly higher than that of bulky CoNbZr $(120 \mu \Omega \mathrm{cm})$. An anisotropy field of around 70 Oe was observed. The anisotropy field was comparatively larger than that of previous studies ${ }^{7), 8}$, which is probably because the CoNbZr film was partly crystallized. However the perpendicular anisotropy that has often been observed for partially crystallized CoNbZr films is not seen for the sample probably because of intensified shape anisotropy of $5 \mathrm{~nm}$ thickness. Fig. 5(b) shows the MH curve of the CoFeB film (45 mm $\times$ $25 \mathrm{~mm}$ and $0.5 \mu \mathrm{m}$ in thickness). The film was deposited by Carousel Sputtering9). Saturation magnetization around $2.2 \mathrm{~T}$ and an anisotropy field $\left(H_{\mathrm{k}}\right)$ of around 260 Oe was observed from the $\mathrm{MH}$ curve.

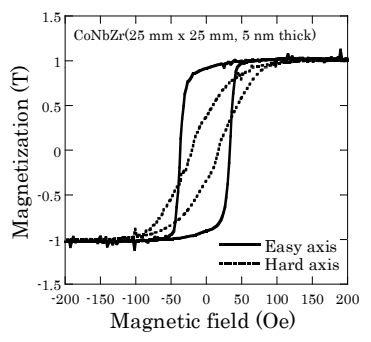

(a) $\mathrm{CoNbZr}$ film

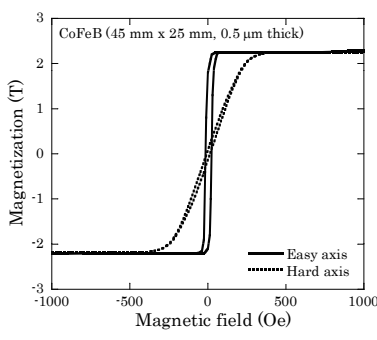

(b) $\mathrm{CoFeB}$ film
Fig. $5 \mathrm{MH}$ curve of the film sample. 


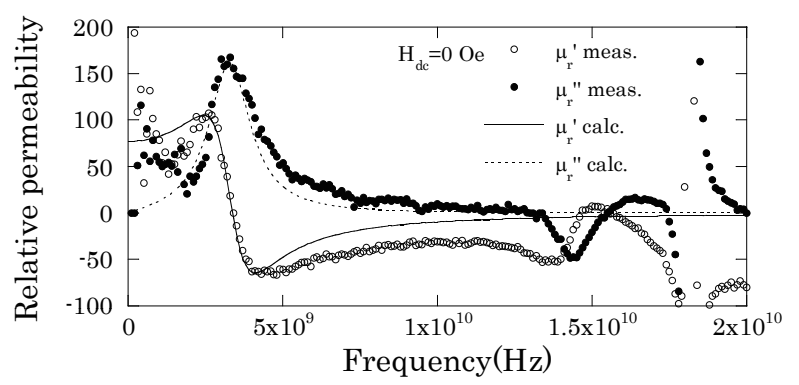

(a) No bias field

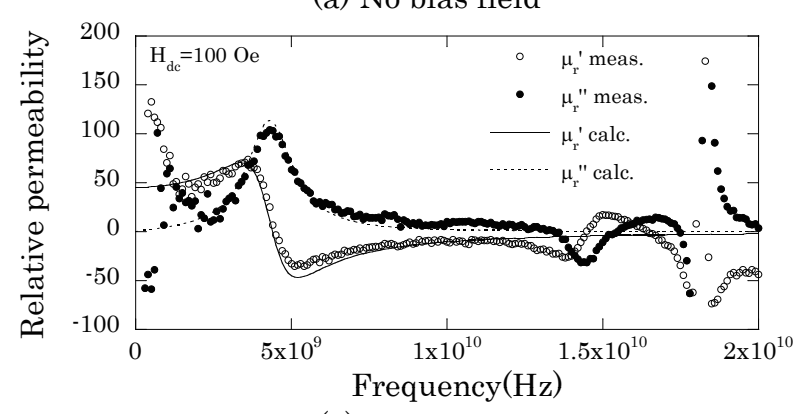

(b) $\mathrm{H}_{\mathrm{dc}}=100 \mathrm{Oe}$

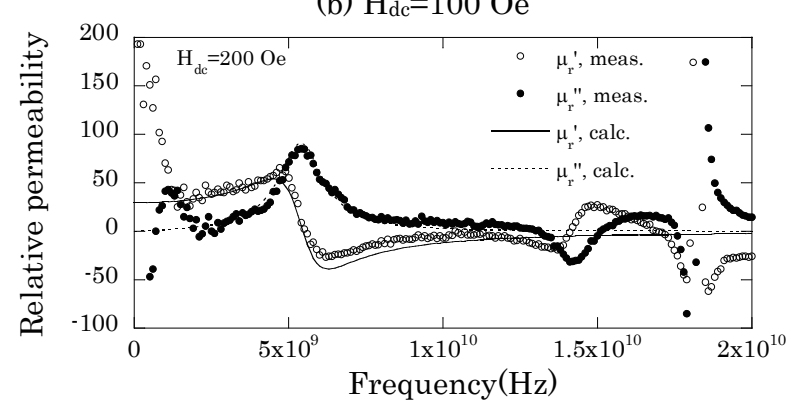

(c) $\mathrm{H}_{\mathrm{dc}}=200 \mathrm{Oe}$

Fig. 6 Relative permeability of CoNbZr film ( $25 \mathrm{~mm} \mathrm{x}$ $25 \mathrm{~mm}, 5 \mathrm{~nm}$ thick).

Fig. 6 shows the hard-axis permeability of amorphous CoNbZr film $(25 \mathrm{~mm} \times 25 \mathrm{~mm}, 5 \mathrm{~nm}$ in thickness $)$ when it was in contact with the flexible probe. Fig. 6 (a) shows the permeability without the bias field, and (b) and (c) show the permeability when bias fields of 100 and 200 Oe, respectively, were applied along the easy axis. The symbols show measured permeability, and the dotted lines and the solid lines show the theoretical permeability based on the Landau-Lifshitz-Gilbert equation and eddy current generation ${ }^{10}$, respectively. A $g$ factor of 2.1311) was used to calculate theoretical permeability. An $\alpha$ (damping factor) of 0.04 was used to fit theoretical permeability to measured spectra. The absolute permeability was calibrated by the application of dc magnetic fields in the direction of the easy axis. The measured permeability roughly corresponded to the theoretical permeability up to $14 \mathrm{GHz}$. The ferromagnetic resonances were observed, and the resonance frequency was found to shift as the bias field increased. The signal to noise ratio in lower frequency decreased and the ferromagnetic resonance frequency was higher than that in the previous report ${ }^{5)}$, which was because the present system consist of magnetic core, therefore small leakage field of the yoke increased the resonance frequency even if the DC current was

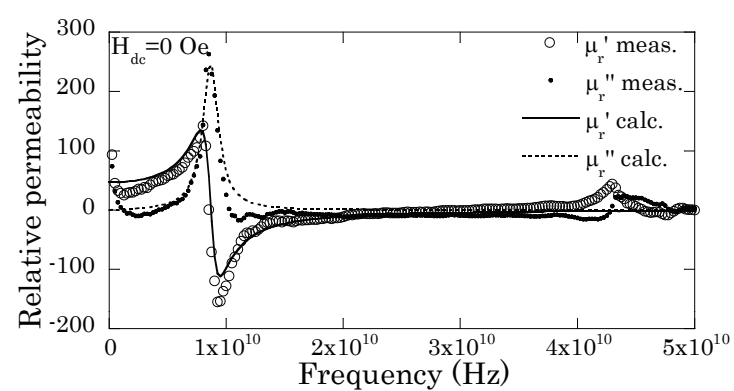

(a) No bias field

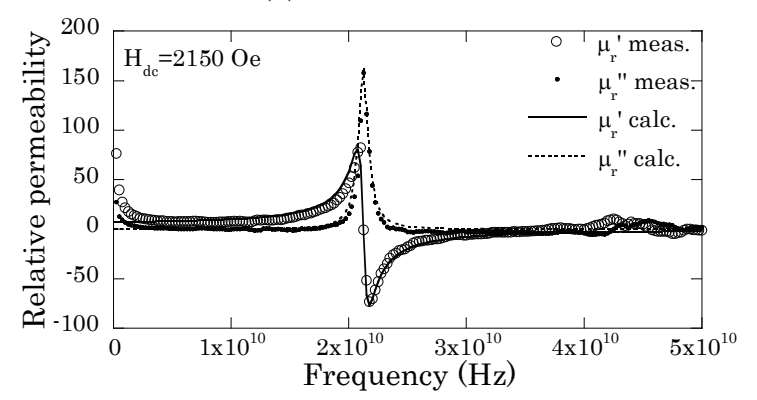

(b) $\mathrm{H}_{\mathrm{dc}}=2150 \mathrm{Oe}$

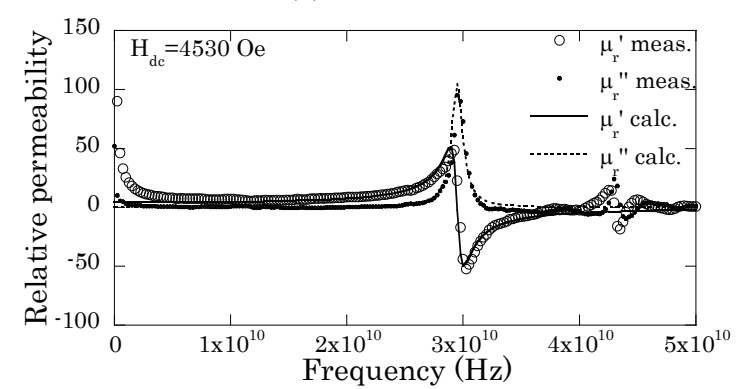

(c) $\mathrm{H}_{\mathrm{dc}}=4530 \mathrm{Oe}$

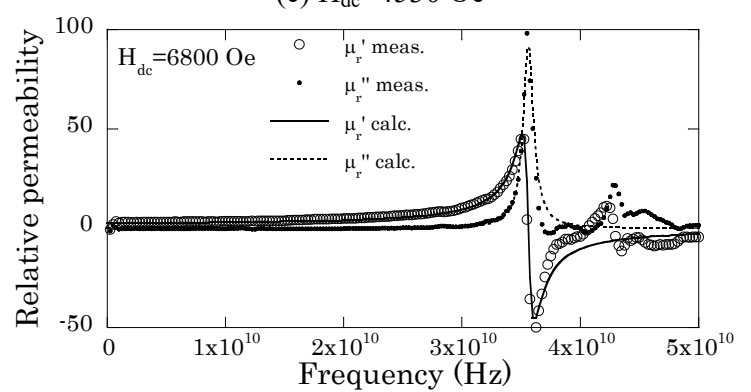

(d) $\mathrm{H}_{\mathrm{dc}}=6800 \mathrm{Oe}$

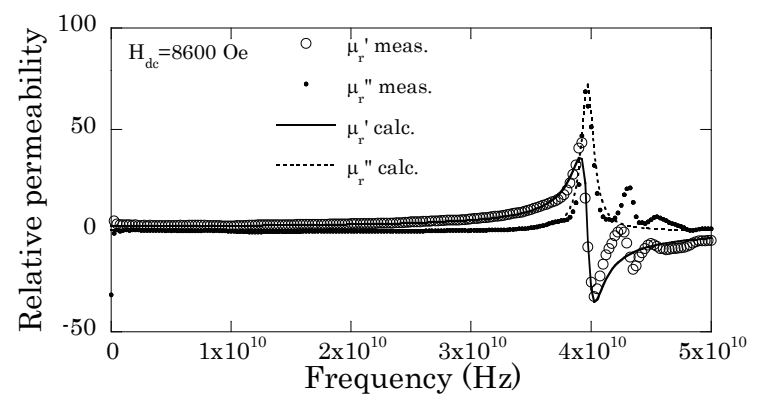

(e) $\mathrm{H}_{\mathrm{dc}}=8600 \mathrm{Oe}$

Fig. 7 Relative permeability of $\mathrm{CoFeB}$ film $(45 \mathrm{~mm} \times$ $25 \mathrm{~mm}, 0.5 \mu \mathrm{m}$ thick).

zero.

Fig. 7 shows the hard-axis permeability of $\mathrm{CoFeB}$ film ( $45 \mathrm{~mm}$ x $25 \mathrm{~mm}, 0.5 \mu \mathrm{m}$ in thickness). Fig. 7 (a) shows the permeability without a bias field, and 
Figs. 7 (b)-(e) show the permeability when bias fields of $2150,4530,6800$, and 8600 Oe were applied along the easy axis. Symbols show measured permeability, and the dotted lines and solid lines show the theoretical permeability based on the Landau-Lifshitz-Gilbert equation and eddy current generation ${ }^{10)}$,respectively. An $\alpha$ (damping factor) of 0.02 and a $g$ factor of 2.13 were used to calculate theoretical permeability. The absolute permeability was calibrated by the application of dc magnetic fields in the direction of the easy axis. The measured permeability was found to roughly correspond to theoretical permeability up to $40 \mathrm{GHz}$. Ferromagnetic resonance shifted from 8 to $40 \mathrm{GHz}$ as the dc field increased. A sharp change was observed around 42-43 GHz. This limit originated from the ferromagnetic resonance of the magnetic film with a strong bias field of about $10.7 \mathrm{kOe}$.

\section{Conclusions}

1. A highly sensitive probe was developed to measure very thin film permeability using a straight microstrip line and a flexible substrate.

2. A CoNbZr film $(25 \mathrm{~mm} \times 25 \mathrm{~mm}, 5 \mathrm{~nm}$ in thickness) was evaluated and the measured permeability was in rough agreement with the theoretical permeability up to $14 \mathrm{GHz}$.

3. A CoFeB film $(45 \mathrm{~mm} \times 25 \mathrm{~mm}, 0.5 \mu \mathrm{m}$ in thickness) with a high anisotropy field was evaluated, and measured permeability was in rough agreement with the theoretical permeability up to $40 \mathrm{GHz}$.

Acknowledgments We would like to thank Prof. Munakata at Sojo University for providing the CoFeB film, Mr. Fujita of Japan Science and Technology Bureau for his advice, Dr. Nakai of Industrial
Technology Institute, Miyagi Prefectural Government for his help in measurement of MH-curves, and the Machine Shop of Tohoku Gakuin University for help in fabricating the probe. This work was supported in part by the Program for Revitalization Promotion of JST.

\section{References}

1) P. A. Calcagno, D. A. Thompson: Rev. Sci. Instrum., 46, 904 (1975).

2) M. Yamaguchi, S. Yabukami and K.I. Arai: IEEE Trans. Magn., 32, 4941 (1996).

3) H. B. Weir: Proc IEEE., 62, 33 (1975).

4) T. Kimura, S. Yabukami, T. Ozawa, Y. Miyazawa, H. Kenju, Y. Shimada: J. Magn. Soc. Jpn. 38, pp. 87-91 (2014).

5) K. Kusunoki, S. Yabukami, T. Ozawa, H. Uetake, H. Yamada, Y. Miyazawa, Y. Shimada： J. Magn. Soc. Jpn. 39, pp. 111-115 (2015).

6) W.H. Press, S.A. Teukolsky, W.T. Vetterling and B.P. Flannery: Numerical Recipes in C (Japanese Edition)., pp.251-281, ( Gijutsu Hyoron sha, Tokyo,1993).

7) H. Katada, T. Shimatsu, I. Watanabe, H. Muraoka, Y. Nakamura and Y. Sugita: J. Magn. Soc. Jpn., 24, 539 (2000).

8) M.L. Schneider, A.B. Kos, and T.J. Silva: Applied Physics Letters, 85, 254 (2004).

9) M. Namikawa, M. Munakata, M. Yagi, M. Motoyama, Y. Shimada, S. Yabukami, M. Yamaguchi, and K.I. Arai : J. Magn. Soc. Jpn., 27, 371 (2003).

10) Y. Shimada, J. Numazawa, Y. Yoneda and A. Hosono: J. Magn. Soc. Jpn., 15, 327(1991).

11) A. Yoshihara, K. Takanashi, M. Shimada, O. Kitakami and Y. Shimada: Jpn. J. Appl. Phys. 33, 3927 (1994).

Received Feb. 10, 2016; Revised Aug. 26, 2016; Accepted Jan. 10, 2017 